\title{
Clearance Concepts: Fundamentals and Application to Pharmacokinetic Behavior of Drugs
}

\author{
Reza Mehvar \\ Department of Biomedical and Pharmaceutical Sciences, School of Pharmacy, Chapman University, Irvine, California, \\ USA.
}

Received, April 13, 2018; Accepted, April 26, 2018; Published, May 21, 2018.

\begin{abstract}
Clearance concepts were introduced into the pharmacokinetics discipline in the 1970s and since then have played a major role in characterization of the pharmacokinetic behavior of drugs. These concepts are based on the relationship between organ extraction ratio or clearance and physiologic parameters such as the organ blood flow and the intrinsic capability of the eliminating organ to remove the free (unbound) drug from the body. Several theoretical models have been developed, which define these relationships and may be used to predict the effects of changes in the physiological parameters on various pharmacokinetic parameters of drugs, such as drug clearance. In this communication, the fundamentals of the two most widely used models of hepatic metabolism, namely the well-stirred (venous equilibrium) and parallel-tube (sinusoidal perfusion) models, are reviewed. Additionally, the assumptions inherent to these models and the differences between them in terms of their predictive behavior are discussed. The effects of changes in the physiologic determinants of clearance on the blood concentration-time profiles of drugs with low and high extraction ratio are also presented using numerical examples. Lastly, interesting and unusual examples from the literature are provided where these concepts have been applied beyond their widely known applications. These examples include estimation of the oral bioavailability of drugs in the absence of otherwise needed intravenous data, differentiation between the role of liver and gut in the first-pass loss of drugs, and distinction between the incomplete absorption and first-pass metabolism in the gastrointestinal tract after the oral administration of drugs. It is concluded that the clearance concepts are a powerful tool in explaining the pharmacokinetics of drugs and predicting the changes in their blood concentration-time courses when the underlying physiologic parameters are altered due to age, disease states, or drug interactions.
\end{abstract}

This article is open to POST-PUBLICATION REVIEW. Registered readers (see "For Readers") may comment by clicking on ABSTRACT on the issue's contents page.

\section{INTRODUCTION}

Clearance concepts were introduced into the pharmacokinetics discipline in the 1970s by Gillette (1), Rowland et al. (2), and Wilkinson and Shand (3). As elegantly described by Benet (4) in a tribute to Dr. Rowland for his contributions in this area, many experimental observations could not be explained by the prevalent pharmacokinetic theories in the late 1960s and early 1970s before the introduction of clearance concepts. In contrast to the heavy reliance on mathematical relationships for description of the pharmacokinetic behavior of drugs in pre-clearance era, the clearance concepts were based on the relationship between organ clearance and physiologic parameters, such as drug free (unbound) fraction in blood $\left(f_{u b}\right)$, intrinsic capability of the eliminating organ to remove the free drug from the body $\left(\mathrm{Cl}^{\prime}\right.$ int $)$, and the organ blood flow $(Q)$. Since the 1970 s, the clearance concepts have been widely used in the literature to explain the pharmacokinetic behavior of many drugs and the effects of changes in the physiological parameters, as a result of disease states, drug-drug interactions, or age, on the blood concentration-time courses of drugs after different routes of administration. The purpose of this communication is to 1) briefly review the fundamental principles of clearance concepts and 2)

\footnotetext{
Corresponding Author: Reza Mehvar, Pharm.D., Ph.D., Department of Biomedical and Pharmaceutical Sciences, School of Pharmacy, Chapman University Rinker Health Science Campus, 9401 Jeronimo Road, Irvine, CA, USA; E-mail: mehvar@chapman.edu
} 
to present examples of the application of clearance concepts in interpretation of pharmacokinetic behavior of drugs.

\section{FUNDAMENTAL CONCEPTS}

\section{Organ Clearance}

The concept of organ clearance and loss of drugs across an organ of elimination have been described in detail previously (5-7). Briefly, the clearance of an eliminating organ $\left(\mathrm{Cl}_{i}\right)$ is the volume of blood cleared of drug by that organ per unit of time, which is defined by the following equation:

$$
C l_{i}=Q_{i} \cdot E_{i}
$$

where $E_{i}$ is the organ extraction ratio or the fraction of the drug, which is eliminated by the organ during one passage, and $Q_{i}$ represents the blood flow to the organ. The $E_{i}$ value may be estimated from the steady state concentration of drug entering $\left(C_{i n}\right)$ and exiting $\left(C_{\text {out }}\right)$ the eliminating organ, as shown in Equation 2:

$$
E_{i}=\frac{C_{\text {in }}-C_{\text {out }}}{C_{\text {in }}}
$$

For hepatic clearance $\left(C_{h}\right)$, Equation 1 may be rewritten in terms of hepatic extraction ratio $\left(E_{h}\right)$ and blood flow $\left(Q_{h}\right)$ :

$$
C l_{h}=Q_{h} \cdot E_{h}
$$

Because the lower and upper limits of $E_{h}$ are zero and 1 , the $C l_{h}$ of drugs could potentially range from zero to $Q_{h}$.

\section{Physiologic Determinants of $\boldsymbol{E}_{h}$ and $\boldsymbol{C l}_{\boldsymbol{h}}$}

In the liver, drugs travel through the sinusoids and come in contact with the hepatocytes, which contain enzymes for drug metabolism. The drug in the sinusoids may be in two forms of protein bound and free. The extent of extraction (and clearance) of drugs depends on at least 3 factors described below.

1. Free fraction of the drug in blood $\left(f_{u b}\right)$ : It is generally assumed that only the free drug may enter the hepatocytes, where it is subject to metabolism or biliary excretion. As a general rule, the higher $f_{u b}$, the larger are $E_{h}$ and $C l_{h}$.

2. Intrinsic clearance of the free drug $\left(\mathrm{Cl}^{\prime}{ }^{\text {int }}\right)$ : This parameter is the intrinsic capability of the liver to remove the drug from the blood in the absence of any flow limitations. In other words, this is the hypothetical volume of blood the liver could clear per unit of time if $Q_{h}$ were unlimited. As a general rule, when $C l^{\prime}{ }_{i n t}$ increases, both $E_{h}$ and $C l_{h}$ will increase.

3. The blood flow $\left(Q_{h}\right): Q_{h}$ affects $E_{h}$ and $C l_{h}$ differently. As the flow increases, the extraction of the drug into the hepatocytes decreases (less time for extraction). However, because $C l_{h}$ is a function of both $E_{h}$ and $Q_{h}$ (Equation 3), overall, an increase in $Q_{h}$ results in an increase (less than proportional) in $C l_{h}$.

\section{Effects of Physiologic Determinants of $E_{h}$ and $C l_{h}$ for High and Low $E_{h}$ Drugs}

The statements above are generally true for any drug with any $E_{h}$. However, the extent by which each of these three parameters $\left(f_{u b}, Q h\right.$, and $\left.C l^{\prime}{ }_{i n t}\right)$ affects $E_{h}$ and $C l_{h}$ is dependent on the initial $E_{h}$ of the drug. For drugs with high metabolic capacity (such as propranolol), $\mathrm{Cl}^{\prime}{ }_{\text {int }}$ may potentially be several-fold higher than the liver blood flow. Therefore, for these drugs, $C l_{h}$ is limited by perfusion $\left(Q_{h}\right)$. Because of high intrinsic metabolic capacity, these drugs have high (close to 1) extraction ratios. At the other extreme, there are drugs for which $\mathrm{Cl}^{\prime}{ }^{\prime}{ }^{n}$ is much less than $Q_{h}$. Therefore, $C l_{h}$ of these drugs is limited by their $\mathrm{Cl}^{\prime}{ }^{\prime}{ }_{\text {int }}$. These drugs have low $E_{h}$ (close to zero).

\section{Models of Hepatic Clearance}

There are different models that may be used to define the relationship among physiologic parameters and hepatic extraction ratio or clearance (8-15). Two of the major models introduced in the 1970s, which are still widely used, are the well-stirred (or venous equilibrium) and parallel tube (or sinusoidal perfusion) models (8-10). These two models are graphically shown in Fig. 1. As shown in the figure, the well-stirred model assumes that the eliminating organ (liver) is a single well-stirred compartment, with the free concentration of the drug in the blood leaving the liver being in equilibrium with and equal to the free drug concentration in the liver water. The parallel-tube model assumes that the liver is composed of identical parallel tubes with even distribution of the enzymes along the length of the tube, and that the blood concentration of free drug decreases exponentially along the length of the tube (Fig. 1). For the parallel-tube model, the average concentration of the drug in the liver is estimated as the logarithmic averages of the inlet $\left(C_{i n}\right)$ and outlet $\left(C_{\text {out }}\right)$ concentrations. 
In both models, the hepatic clearance $\left(\mathrm{Cl}_{h}\right)$ is related to the hepatic blood flow $\left(Q_{h}\right)$ and the extraction ratio $\left(E_{h}\right)$ by Equation 3. Additionally, in both models, hepatic availability $\left(F_{h}\right)$, which is defined by the $\frac{C_{\text {out }}}{C_{\text {in }}}$ ratio, is related to $E_{h}$ using the following equation:

$$
F_{h}=1-E_{h}
$$

As demonstrated in Equation $4, F_{h}$ is the fraction of the drug escaping extraction by the liver. In other words, it is the fraction of the concentration entering the liver, which exits the liver intact.

\section{Well-Stirred Model}

For the well-stirred model, the relationships between $E_{h}$ or $F_{h}$ and $f_{u b}, C l$ 'int, and $Q_{h}$ are defined by the following equations:

$$
E_{h}=\frac{f_{u b} \cdot C l_{i n t}^{\prime}}{Q_{h}+f_{u b} \cdot C l_{i n t}^{\prime}}
$$

$$
F_{h}=1-E_{h}=1-\frac{f_{u b} \cdot C l_{i n t}^{\prime}}{Q_{h}+f_{u b} \cdot C l_{i n t}^{\prime}}=\frac{Q_{h}}{Q_{h}+f_{u b} \cdot C l_{i n t}^{\prime}}
$$

Therefore, based on the well-stirred model, $\mathrm{Cl}_{h}$ may be defined in terms of its individual components using the following equation:

$$
C l_{h}=Q_{h} \times \frac{f_{u b} \cdot C l_{\text {int }}^{\prime}}{Q_{h}+f_{u b} \cdot C l_{\text {int }}^{\prime}}
$$

The $C l_{\text {int }}^{\prime}$ parameter, which is an indication of the intrinsic capability of the liver to remove the drug from the body in the absence of any flow limitation, is related to the sum of activities of the enzymes that metabolize the drug:

$$
C l_{\text {int }}^{\prime}=\sum_{i=1}^{n} \frac{V_{\max , i}}{K_{m, i}+C_{L, u}}
$$

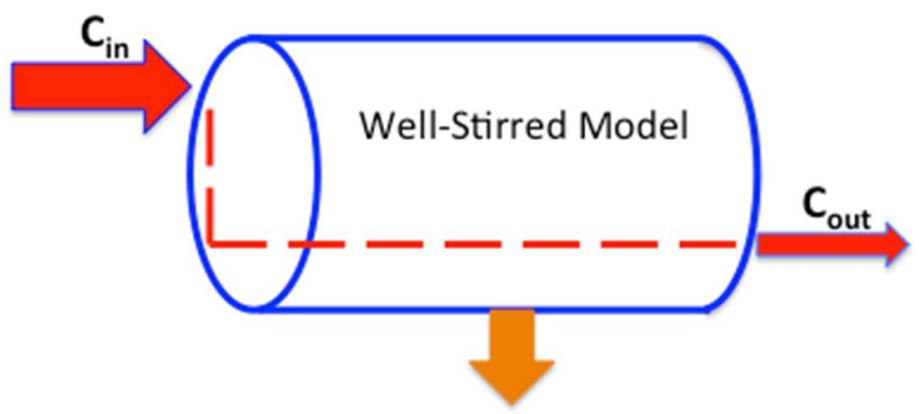

Elimination

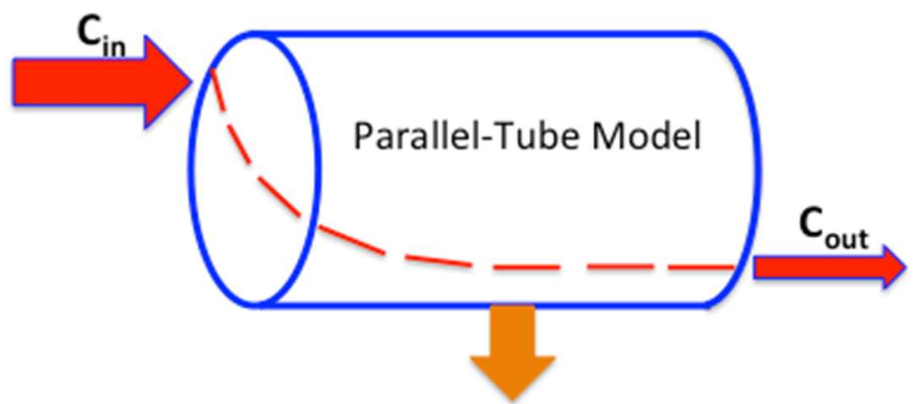

Elimination

Figure 1. Graphical representations of the well-stirred (top) and parallel-tube (bottom) models of organ clearance. $C_{i n}$ and $C_{o u t}$ represent concentration of the drug entering and leaving the liver, respectively, and the dashed lines indicate the unbound concentration of the drug in the liver along the direction of flow. Adapted from reference (8). 
In the above equation, $V_{\max , i}, K_{m, i}$, and $C_{L, u}$ refer to the maximum velocity and Michaelis-Menten constant of the individual enzymes involved in the metabolism of the drug and the unbound concentration of the drug in the liver, respectively. If the drug is also subject to biliary excretion, $\mathrm{Cl}^{\prime}{ }_{i n t}$ is the summation of both metabolic and excretory intrinsic clearances.

For simplicity, some investigators use $C l_{\text {int }}$, instead of the term $f_{u b} \cdot C l_{\text {int }}^{\prime}$, in Equations 5, 6 and 7:

$$
\begin{gathered}
E_{h}=\frac{C l_{i n t}}{Q_{h}+C l_{i n t}} \\
F_{h}=\frac{Q_{h}}{Q_{h}+C l_{i n t}} \\
C l_{h}=Q_{h} \times \frac{C l_{i n t}}{Q_{h}+C l_{i n t}}
\end{gathered}
$$

The term $C l_{\text {int }}$ is defined as the intrinsic clearance of the total (free plus unbound) drug. Equation 9 may be rearranged to estimate $C l_{\text {int }}$ when the $E_{h}$ and $Q_{h}$ values are known:

$$
C l_{\text {int }}=\frac{E_{h} \times Q_{h}}{1-E_{h}}=\frac{E_{h} \times Q_{h}}{F_{h}}
$$

For drugs with high $E_{h}$ (i.e., high values of $C l_{\text {int }}$ relative to $Q_{h}$ ), Equations 9,10 , and 11 would simplify to the following equations:

$$
\begin{gathered}
E_{h}=\frac{C l_{i n t}}{Q_{h}\left(\ll C l_{i n t}\right)+C l_{i n t}} \approx \frac{C l_{i n t}}{C l_{\text {int }}} \approx 1 \\
F_{h}=\frac{Q_{h}}{Q_{h}\left(\ll C l_{i n t}\right)+C l_{i n t}} \approx \frac{Q_{h}}{C l_{i n t}} \\
C l_{h}=Q_{h} \times \frac{C l_{i n t}}{Q_{h}\left(\ll C l_{i n t}\right)+C l_{i n t}} \approx Q_{h} \times \frac{C l_{i n t}}{C l_{i n t}} \approx Q_{h}
\end{gathered}
$$

These approximate equations indicate that for drugs with very high $E_{\mathrm{h}}$ (close to 1 ), $F_{h}$ is proportionally related to $Q_{h}$ and inversely related to $C l_{\text {int }}$. Additionally, the $\mathrm{Cl}_{h}$ of these drugs is perfusionlimited. Examples of drugs with high $E_{h}$ include propranolol, verapamil, morphine, meperidine, nitroglycerine, and lidocaine.

On the other hand, for drugs with low $E_{h}$ (i.e., low values of $C l_{\text {int }}$ relative to $\left.Q_{h}\right), E_{h}$ is directly related to $C l_{\text {int }}$ and inversely related $Q_{h}, F_{h}$ is almost close to 1 , and $C l_{h}$ of the drug becomes almost equal to $C l_{\text {int }}$ or $f_{u b} \cdot C l_{\text {int }}^{\prime}$ :

$$
\begin{gathered}
E_{h}=\frac{C l_{\text {int }}}{Q_{h}+C l_{\text {int }}\left(\ll Q_{h}\right)} \approx \frac{C l_{\text {int }}}{Q_{h}} \\
F_{h}=\frac{Q_{h}}{Q_{h}+C l_{\text {int }}\left(\ll Q_{h}\right)} \approx \frac{Q_{h}}{Q_{h}} \approx 1 \\
C l_{h}=Q_{h} \times \frac{C l_{\text {int }}}{Q_{h}+C{ }_{\text {int }}\left(\ll Q_{h}\right)} \approx Q_{h} \times \frac{C l_{\text {int }}}{Q_{h}} \approx C l_{\text {int }} \approx \\
f_{u b} \cdot C l_{\text {int }}^{\prime}
\end{gathered}
$$

Examples of drugs with low $E_{h}$ include warfarin, tolbutamide, diazepam, erythromycin, and theophylline.

\section{Parallel-Tube Model}

For the parallel-tube model, the relationships between $E_{h}, F_{h}$, or $C l_{h}$ and $f_{u b}, C l$ ' $i n t$, and $Q_{h}$ are given below:

$$
\begin{gathered}
E_{h}=1-e^{\frac{-f_{u b} \cdot C l_{\text {int }}^{\prime}}{Q_{h}}}=1-e^{\frac{-C l_{\text {int }}}{Q_{h}}} \\
F_{h}=1-E_{h}=e^{\frac{-f_{u b} \cdot C l_{\text {int }}^{\prime}}{Q_{h}}}=e^{\frac{-C l_{\text {int }}}{Q_{h}}} \\
C l_{h}=Q_{h} \cdot E_{h}=Q_{h}\left(1-e^{\frac{-f_{u b} \cdot C l_{\text {int }}^{\prime}}{Q_{h}}}\right)=Q_{h}(1- \\
\left.e^{\frac{-C l_{\text {int }}}{Q_{h}}}\right)
\end{gathered}
$$

If Equation 19 or Equation 20 for the parallel tube model is to be used for the estimation of $C l_{\text {int }}$ when $E_{h}$ and $Q_{h}$ are known, this may be done by taking the natural logarithm of the exponential term:

$$
\begin{gathered}
\ln \left(1-E_{h}\right)=\frac{-C l_{\text {int }}}{Q_{h}} \\
C l_{\text {int }}=-Q_{h} \times \ln \left(1-E_{h}\right)=-Q_{h} \times \ln F_{h}
\end{gathered}
$$

Similar to the well-stirred model, for drugs with the very high or very low $E_{h}$, Equation 21 predicts that the $C l_{h}$ is limited by $Q_{h}$ or $C l_{\text {int }}\left(f_{u b} \cdot C l_{\text {int }}^{\prime}\right)$, respectively.

For both models, the above equations assume that the permeability of the drug across the interstitial space and hepatocyte membranes is much higher than the $\mathrm{Cl}^{\prime}{ }_{\text {int }}$ of the drug. Therefore, permeability is not the rate-limiting step in the elimination of the drug. However, if permeability becomes an issue, these equations need to be modified to account for the permeability parameters. Pravastatin is an 
example of drugs with uptake permeability-limited elimination (16).

\section{Differences between the Models in Predictions of Pharmacokinetics of Drugs}

Simulations have shown (8) that the two models behave relatively close to each other with regard to the effects of changes in $f_{u b}$ and/or $C l^{\text {int }}$ (i.e., $C l_{\text {int }}$ ). However, the major difference between the two models is with regard to their prediction of hepatic availability $\left(F_{h}\right)$ based on changes in $Q_{h}$ for the high $E_{h}$ dugs. Although the differences between the two models for the prediction of $E_{h}$ even at high $E_{h}$ is relatively small $(30 \%)$, this translates to very large differences between the two models in prediction of $F_{h}$ and oral AUC of drugs with high hepatic $E_{h}(8)$. This is because at high $E_{h}, F_{h}$ changes linearly relative to $Q_{h}$ for the well-stirred model as demonstrated in Equation $14\left(F_{h} \approx Q_{h} / C l_{\text {int }}\right)$. However, for the parallel-tube model, $F_{h}$ changes exponentially relative to $Q_{h}$, as demonstrated in Equation $20\left(F_{h}=e^{-C l_{\text {int }} / Q_{h}}\right)$. Therefore, at the extreme $E_{h}$ value of 0.99 , the difference in prediction of $F_{h}$ for the two models may be over a thousand-fold (8). Further experimental studies $(9,10)$ have suggested that for most drugs, such as lidocaine, the well-stirred model predicts the clearance of the drug better. Therefore, most investigators use the wellstirred model in their analysis of hepatic clearance of drugs, unless it is proven to be not applicable. However, it has recently been argued that the studies designed to distinguish between the two models are inherently biased towards the well-stirred model (17), a notion that is subject to debate (18) and outside the scope of this review. Because of the prevalence of the use of the well-stirred model in the literature, we will use this model for the remainder of our discussion and case studies presented here.

\section{The Relationship between Hepatic Availability $\left(F_{h}\right)$ and Oral Bioavailability $(F)$}

Figure 2 depicts the potential barriers for an oral dose to reach systemic circulation, which may result in the loss of drugs after their oral administration (19). After the intravenous dosing, however, the drug is introduced directly into the systemic circulation. Therefore, the oral bioavailability of drugs $(F)$, or the extent by which an oral dose enters the systemic circulation, is defined by the ratio of the blood AUCs after the oral and intravenous administration. As demonstrated in Fig. 2, after oral administration, the drug is absorbed from the gastrointestinal tract and passes through the liver before reaching the systemic circulation. This means the orally-administered drug entering the systemic circulation has to pass through the liver first ("first-pass effect"). This is different from the intravenous administration, where the drug is directly introduced into the systemic circulation without passing through the liver first (Fig. 2). Obviously, in both cases (intravenous and oral), after reaching the systemic circulation, a fraction of the drug will be passing through different organs, including the liver, with each circulation. However, the oral drug has one extra "pass" through the liver during the absorption process. Therefore, the oral bioavailability of drugs $(F)$ is influenced by their hepatic availability $\left(F_{h}\right)$. In addition to $F_{h}, F$ is also affected by the loss of drug during the absorption process such as incomplete absorption and/or metabolism/degradation in the gastrointestinal tract (Fig. 2). To clarify this, consider an example where $200 \mathrm{mg}$ of a drug is administered orally to a patient. Assume only $40 \%$ of the dose is absorbed orally (fraction of the dose absorbed or $F_{a b}$ of 0.4 ), which means only $80 \mathrm{mg}$ reaches the enterocytes. If the drug is subject to metabolism in the gut, resulting in $50 \%$ of the drug escaping metabolism in the enterocytes $\left(F_{g}\right.$ of 0.5$)$, only $40 \mathrm{mg}$ of the drug reaches the liver. Assuming a hepatic $E_{h}$ of $0.7\left(F_{h}\right.$ or hepatic availability of 0.3$), 30 \%$ of the drug reaching the liver will pass intact into the systemic circulation $(0.3 \times 40 \mathrm{mg}$ or $12 \mathrm{mg})$. This means that out of the 200 -mg oral dose, only $12 \mathrm{mg}$ reaches the systemic circulation. Therefore, the oral bioavailability of this drug (relative to intravenous administration), which may be estimated experimentally by the oral AUC: intravenous AUC ratio, is only 0.06 or $6 \%(12 / 200)$. The following equation describes the relationship among $F, F_{a b}, F_{g}$, and $F_{h}$ :

$$
\begin{gathered}
F=F_{a b} \times F_{g} \times F_{h} \\
F=0.4 \times 0.5 \times 0.3=0.06
\end{gathered}
$$

It should be noted that if the absorption of the drug is complete and there is no gastrointestinal metabolism (i.e., $F_{a b} \times F_{g}=1$ ), the hepatic availability and oral bioavailability would be the same. In other words, the oral bioavailability of drugs cannot be more than their hepatic availability. 


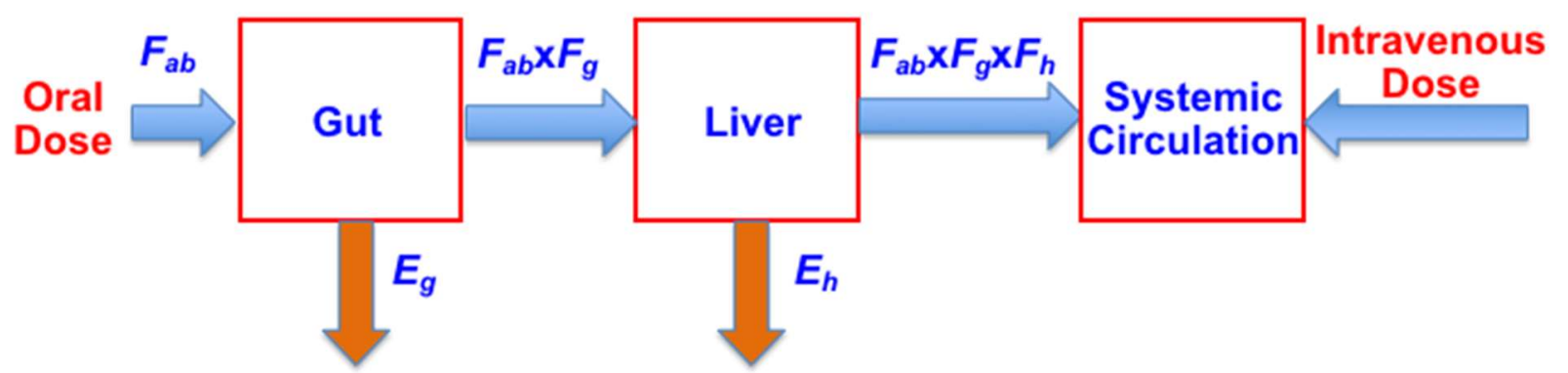

Figure 2. Barriers to drug entry into the systemic circulation after oral administration of drugs. For comparison, direct introduction of the drug into the systemic circulation after intravenous administration is also shown. Abbreviations: $F_{a b}$, fraction of the dose absorbed; $F_{g}$, fraction of the dose escaping gut metabolism; $F_{h}$, fraction of the dose escaping liver metabolism; $E_{g}$, gut extraction ratio; $E_{h}$, liver extraction ratio. Adapted from reference (19).

\section{Effects of $Q_{h}, f_{u b}$, and $C l^{\prime}{ }_{i n t}$ on the Blood Concentration-Time Courses of Low and High $E_{h}$ Drugs}

After intravenous dosing, the only pharmacokinetic parameter that affects the AUC of a drug is its systemic $\mathrm{Cl}$. However, after oral dosing, the AUC is dependent on both the systemic $\mathrm{Cl}$ and oral availability $(F)$, both of which are subject to change if $f_{u b}, C l$ 'int, and/or $Q_{h}$ is changed. Here, we examine the effects of a change in the determinants of $C l_{h}$ of drugs on their blood concentration-time profiles for the low and high $E_{h}$ drugs. For simplicity, we assume that the drug is eliminated by hepatic metabolism only, which means the systemic $C l$ is equal to $C l_{h}$. Additionally, we assume that the oral absorption of the drug is complete with no metabolism in the gut, which means the oral availability of the drug $(F)$ is equal to its hepatic availability $\left(F_{h}\right)$.

\section{Low Extraction Ratio Drugs}

The characteristics of a hypothetical drug with a very low $E_{h}$ value of 0.0011 under normal conditions and when the $\mathrm{Cl}_{\text {int }}\left(\mathrm{Cl}^{\prime}\right.$ int or fub) or $Q_{h}$ values are increased by a factor of 2 are listed in Table 1. Additionally, the corresponding blood concentration-time courses of the drug are presented in Fig. 3. After selection of $\mathrm{Cl}^{\prime}{ }^{\prime}{ }^{\prime}$ and $f_{u b}$ values for this drug to generate a low $C l_{\text {int }}$ value $(0.1 \mathrm{~L} / \mathrm{h})$ relative to the hepatic blood flow $(90 \mathrm{~L} / \mathrm{h})$, the $E_{h}, F_{h}$, and $\mathrm{Cl}_{h}$ are calculated using Equations 9, 4, and 3, respectively. As stated above, for a low $E_{h}$ drug, $C l_{h}$ is expected to be close to $C l_{\text {int }}$ or $f_{u b} \cdot C l_{\text {int }}^{\prime}$. This is also shown in Table 1 for Control subjects, where the $\mathrm{Cl}_{\text {int }}$ and $\mathrm{Cl}_{h}$ values are almost identical for this hypothetical drug. Additionally, $F_{h}$ for this drug is very high (almost 1) (Table 1). Assuming a volume of distribution of $5 \mathrm{~L}$, the half-life of the drug is $35 \mathrm{~h}$ in Control subjects (Table 1). As demonstrated in Table 1 , the changes in either $C l$ 'int or $f_{u b}$ would produce the same net effect on the other kinetic parameters of the drug because they are both reflected in the $C l_{\text {int }}$ value. A two-fold increase in $C l^{\prime}{ }_{i n t}$ or $f_{u b}$ results in a two-fold increase in $C l_{i n t}, E_{h}$, and $C l_{h}$ for this low $E_{h}$ drug (Table 1). Additionally, a two-fold increase in $\mathrm{Cl}_{h}$ also results in a two-fold decrease in the half-life of the drug (Table 1). However, the $F_{h}$ value of the drug remains close to 1 , regardless of the changes in $f_{u b}$ or $\mathrm{Cl}^{\prime}{ }_{\text {int }}$ (Table 1). As demonstrated in Figs. 3A and 3B, a two-fold increase in $C l_{\text {int }}$ for a low $E_{h}$ drug results in an almost two-fold increase in its $\mathrm{Cl}$, which is demonstrated by a corresponding two-fold decrease in the AUC and half-life after both intravenous and oral dosing. An example of such change is the effects of coadministration of warfarin, a low $E_{h}$ drug, with rifampin, which induces the metabolism of warfarin (higher $C l{ }^{\prime}{ }_{i n t}$ ), resulting in a higher clearance, lower AUC, and a shorter half-life (20).

As for $Q_{h}$, neither intravenous nor oral profile of a low $E_{h}$ drug is affected by a change in $Q_{h}$ (Figs. 3C and 3D). Although a two-fold increase in $Q_{h}$ results in a two-fold decrease in $E_{h}$, the $C l_{h}$ value remains unchanged because the decrease in $E_{h}$ is compensated by a corresponding increase in $Q_{h}$ (Table 1). This is consistent with the notion that the $\mathrm{Cl}$ of a low $E_{h}$ drugs is mostly dependent on $f_{u b}$ and $C l{ }^{\prime}{ }_{i n t}$ (Equation 18). Additionally, $F_{h}$ of the drug is very high regardless of the change in $Q_{h}$ (Table 1). 
(A)

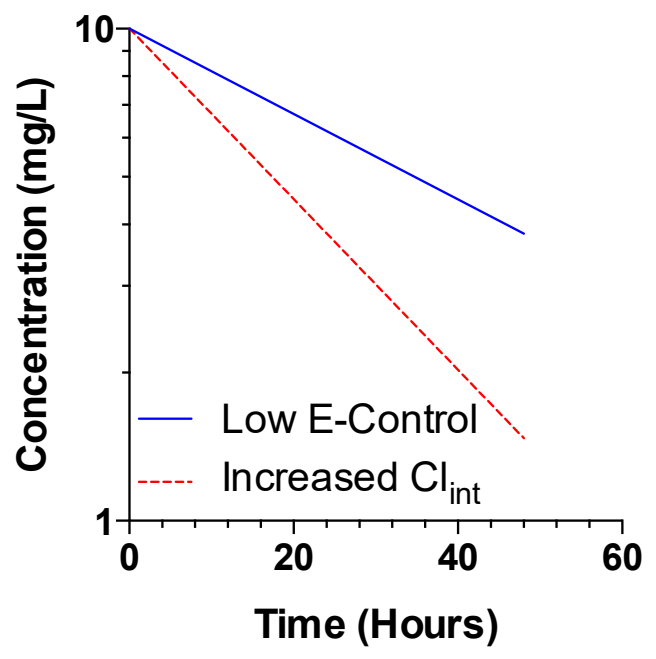

(C)

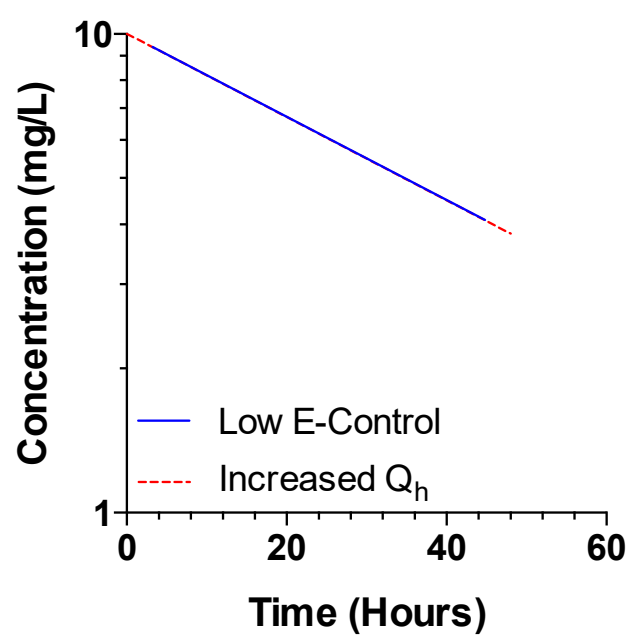

(B)

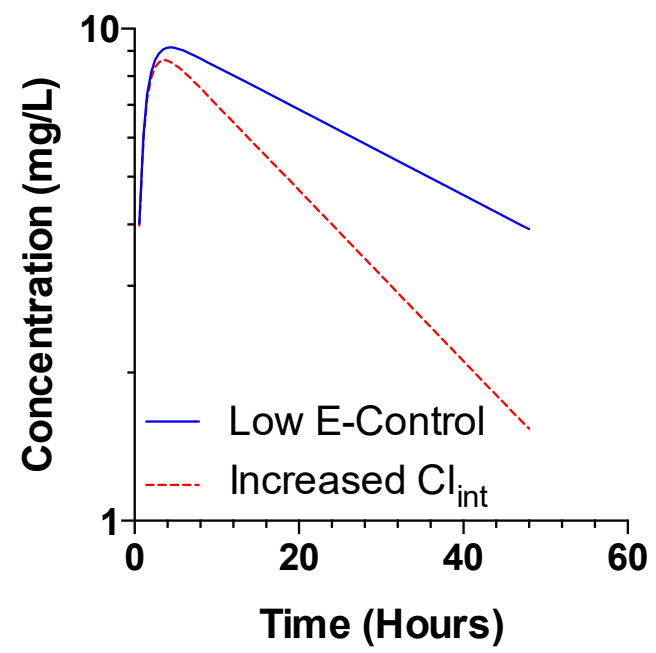

(D)

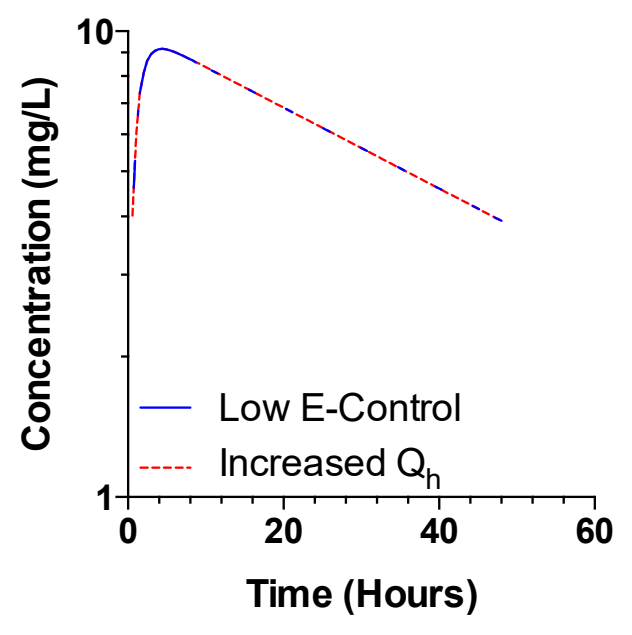

Figure 3. Effects of a two-fold increase in $C l_{i n t}\left(f_{u b}\right.$ or $C l^{\prime}$ int $)$ (top panels) or $Q_{h}$ (bottom panels) on the blood concentrationtime courses of a hypothetical low $E_{h}$ drug after intravenous (left) or oral (right) administration of a 50-mg dose of the drug. The kinetic parameters are listed in Table 1.

Table 1. The pharmacokinetic parameters of a hypothetical drug a with low hepatic extraction ratio $\left(E_{h}\right)$ in the absence (Control) and presence of changes in the intrinsic capability of the liver to remove the free $\operatorname{drug}\left(\mathrm{Cl}^{\prime}{ }_{\text {int }}\right)$, free drug fraction in blood $\left(f_{u b}\right)$, or hepatic blood flow $\left(Q_{h}\right)$.

\begin{tabular}{|c|c|c|c|c|c|c|c|c|c|}
\hline Condition & $\begin{array}{l}C l^{\prime} \text { int } \\
(\mathrm{L} / \mathrm{h})\end{array}$ & $f_{u b}$ & $\begin{array}{c}C_{\text {int }} \\
(\mathrm{L} / \mathrm{h})\end{array}$ & $\underset{(\mathbf{L} / \mathbf{h})}{Q_{h}}$ & $\boldsymbol{E}_{\boldsymbol{h}}^{\mathrm{b}}$ & $\boldsymbol{F}_{\boldsymbol{h}}^{\mathrm{b}}$ & $\begin{array}{c}C l_{h}{ }^{\mathrm{b}} \\
(\mathrm{L} / \mathrm{h})\end{array}$ & $\begin{array}{c}V \\
(L)\end{array}$ & $\begin{array}{l}t_{1 / 2}{ }^{\mathrm{c}} \\
\text { (h) }\end{array}$ \\
\hline Control & 10 & 0.01 & 0.10 & 90 & 0.00111 & 0.999 & 0.0999 & 5 & 34.7 \\
\hline$\uparrow \mathrm{Cl}^{\prime}{ }_{\text {int }} \times 2$ & 20 & 0.01 & 0.20 & 90 & 0.00222 & 0.998 & 0.200 & 5 & 17.4 \\
\hline$\uparrow f_{u b} \times 2$ & 10 & 0.02 & 0.20 & 90 & 0.00222 & 0.998 & 0.200 & 5 & 17.4 \\
\hline$\uparrow Q_{h} \times 2$ & 10 & 0.01 & 0.10 & 180 & 0.000555 & 0.999 & 0.0999 & 5 & 34.7 \\
\hline
\end{tabular}

${ }^{a}$ The hypothetical drug has a $C l$ ' ${ }_{\text {int }}$ of $10 \mathrm{~L} / \mathrm{h}$, a $f_{u b}$ of 0.01 , and a volume of distribution (V) of $5 \mathrm{~L}$ under baseline conditions, with elimination through the liver only.

${ }^{\mathrm{b}} E_{h}, F_{h}$, and $C l_{h}$ were estimated from the values of $C l$ ' ${ }_{i n t}, f_{u b}$, and $Q_{h}$ using Equations 9, 4, and 3, respectively.

${ }^{\mathrm{c}}$ Half-life $\left(t_{l / 2}\right)$ is estimated from $t_{1 / 2}=\frac{0.693 \times V}{C l}$, where $C l$ is assumed to be equal to $C l_{h}$. 


\section{High Extraction Ratio Drugs}

The pharmacokinetic characteristics of a high $E_{h}$ drug in the absence (Control) or presence of changes in $C l^{\prime}{ }_{i n t}, f_{u b}$, or $Q_{h}$ are listed in Table 2. Additionally, the corresponding blood concentration-time courses are shown in Fig. 4. The $C l_{\text {int }}$ of the drug in Control subjects $(1500 \mathrm{~L} / \mathrm{h})$ is much more than the $Q_{h}$ of 90 $\mathrm{L} / \mathrm{h}$, hence a high $E_{h}$ of 0.943 (Table 2). As expected, the drug has a very low $F_{h}$ value of 0.0566 . Additionally, assuming a V of $400 \mathrm{~L}$, the drug has a half-life of $3.26 \mathrm{~h}$ (Table 2). In contrast to the low $E_{h}$ drug (Table 1), doubling the $\mathrm{Cl}_{\text {int }}$ by doubling either $f_{u b}$ or $C l$ 'int does not substantially affect the $E_{h}, C l_{h}$, or half-life of this high $E_{h}$ drug (Table 2). The lack of changes in the $C l_{h}$ of this high $E_{h}$ drug after doubling $C l_{\text {int }}$ is predictable because the $C l_{h}$ of these drugs are mostly perfusion limited. However, the hepatic availability (and oral bioavailability) of the drug is reduced by almost $50 \%$ when $C l_{\text {int }}$ is increased by a factor of 2 (Table 2). This is because after oral dosing, the $F_{h}$ of the high $E_{h}$ drugs is almost linearly (inversely) related to their $\mathrm{Cl}_{\text {int }}$ (Equation 14). Consequently, whereas the blood concentration-time courses of the high $E_{h}$ drug after the intravenous administration are insensitive to changes in $C_{\text {int }}$ (no change in $C l$ ) (Fig. 4A), the $F_{h}$, blood concentrations, and $\mathrm{AUC}$ of the drug after the oral administration are reduced by an almost $50 \%$ in the presence of a twofold increase in $\mathrm{Cl}_{\text {int }}$ (Fig. 4B).

The above discussion clearly shows that the disease states and/or drug interactions that affect $\mathrm{Cl}^{\prime}{ }_{\text {int }}$ and/or $f_{u b}$ would be reflected in the blood concentration-time courses of high $E_{h}$ drugs only after the oral dosing, but not after the intravenous administration (Figs. 4A and 4B).

An example of such effect is the interaction between the enzyme inducer pentobarbital and the high $E_{h}$ drug alprenolol reported by Alvan et al. (21).
In that study, a single dose of alprenolol was administered intravenously or orally before and after 10-14 daily doses of pentobarbital. Whereas pentobarbital reduced the AUC of the orallyadministered alprenolol by more than $75 \%$ without affecting the alprenolol's half-life, it did not significantly affect the AUC or half-life of the drug after its intravenous administration.

As for $Q_{h}$, in contrast to the low $E_{h}$ drugs, the pharmacokinetics of high $E_{h}$ drugs are affected by changes in this parameter after both intravenous and oral administration. As shown in Table 2, doubling $Q_{h}$ hardly affects the $E_{h}$ value but almost doubles the $F_{h}$ and $\mathrm{Cl}_{h}$ of the drug. Therefore, the blood concentration-time course of the drug after the intravenous administration shows a $50 \%$ reduction in the AUC and half-life (Fig. 4C). The oral profile would be affected by changes in both $C l$ and $F_{h}$ (Fig. 4D). After oral dosing, an increase in $\mathrm{Cl}$ due to an increase in $Q_{h}$ causes a proportional reduction in AUC and shortening of half-life. However, an increase in $Q_{h}$ also proportionally increases $F_{h}\left(F_{h} \approx\right.$ $\left.\frac{Q_{h}}{C l_{\text {int }}}\right)$ and AUC. Therefore, as demonstrated in Fig. $4 \mathrm{D}$, the AUC after oral dosing remains the same. Please note that the above discussions are based on the well-stirred model, which predicts that oral AUC is not dependent on $Q_{h}$ for drugs that are eliminated by hepatic metabolism:

$$
A U C_{P O}=\frac{F \cdot D o s e}{C l}
$$

In the above equation, an increase in $Q_{h}$ causes proportional increases in both $\mathrm{Cl}$ and $\mathrm{F}$, without any significant changes in the AUC for a drug with high $E_{h}$.

Table 2. The pharmacokinetic parameters of a hypothetical drug ${ }^{\mathrm{a}}$ with high hepatic extraction ratio $\left(E_{h}\right)$ in the absence (Control) and presence of changes in the intrinsic capability of the liver to remove the free $\operatorname{drug}\left(\mathrm{Cl}^{\prime}{ }_{\text {int }}\right)$, free drug fraction in blood $\left(f_{u b}\right)$, or hepatic blood flow $\left(Q_{h}\right)$

\begin{tabular}{|c|c|c|c|c|c|c|c|c|c|}
\hline Condition & $\begin{array}{l}\mathrm{Cl}^{\prime}{ }_{i n t} \\
(\mathrm{~L} / \mathrm{h})\end{array}$ & $f_{u b}$ & $C l_{\text {int }}(\mathrm{L} / \mathrm{h})$ & $\begin{array}{c}Q_{h} \\
(\mathbf{L} / \mathbf{h})\end{array}$ & $\overline{E_{h}{ }^{\mathrm{b}}}$ & $\boldsymbol{F}_{\boldsymbol{h}}^{\mathrm{b}}$ & $\begin{array}{c}C l_{h}{ }^{b} \\
(\mathrm{~L} / \mathrm{h})\end{array}$ & $\begin{array}{c}V \\
(L)\end{array}$ & $\begin{array}{l}t_{1 / 2}{ }^{c} \\
\text { (h) }\end{array}$ \\
\hline Control & 15000 & 0.1 & 1500 & 90 & 0.943 & 0.0566 & 84.9 & 400 & 3.26 \\
\hline$\uparrow \mathrm{Cl}^{\prime}{ }_{i n t} \mathrm{x} 2$ & 30000 & 0.1 & 3000 & 90 & 0.971 & 0.0291 & 87.4 & 400 & 3.17 \\
\hline$\uparrow f_{u b} \times 2$ & 15000 & 0.2 & 3000 & 90 & 0.971 & 0.0291 & 87.4 & 400 & 3.17 \\
\hline$\uparrow Q_{h} \times 2$ & 15000 & 0.1 & 1500 & 180 & 0.893 & 0.107 & 161 & 400 & 1.72 \\
\hline
\end{tabular}

${ }^{\mathrm{a}}$ The hypothetical drug has a $C l$ ' ${ }_{\text {int }}$ of $15000 \mathrm{~L} / \mathrm{h}$, a $f_{u b}$ of 0.1 , and a volume of distribution (V) of $400 \mathrm{~L}$ under baseline conditions, with elimination through the liver only.

${ }^{\mathrm{b}} E_{h}, F_{h}$, and $C l_{h}$ were estimated from the values of $C l$ ' ${ }_{i n t}, f_{u b}$, and $Q_{h}$ using Equations 9, 4, and 3, respectively.

${ }^{\mathrm{c}}$ Half-life $\left(t_{l / 2}\right)$ is estimated from $t_{1 / 2}=\frac{0.693 \times V}{C l}$, where $C l$ is assumed to be equal to $C l_{h}$. 
(A)

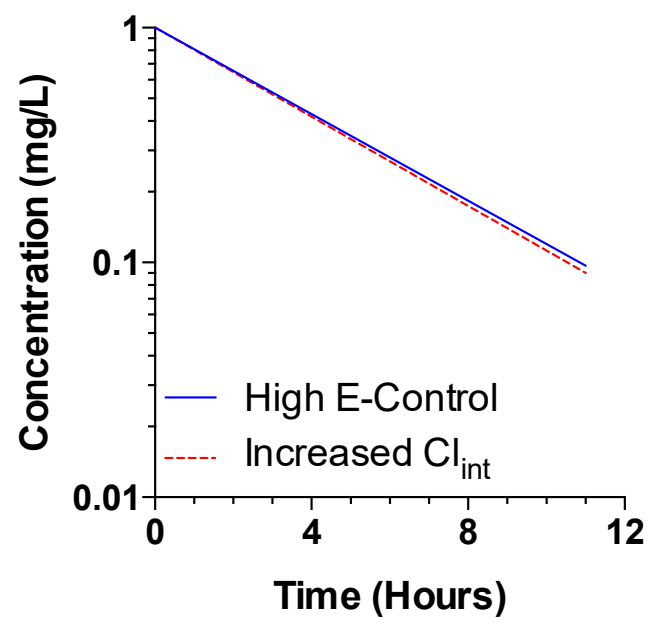

(C)

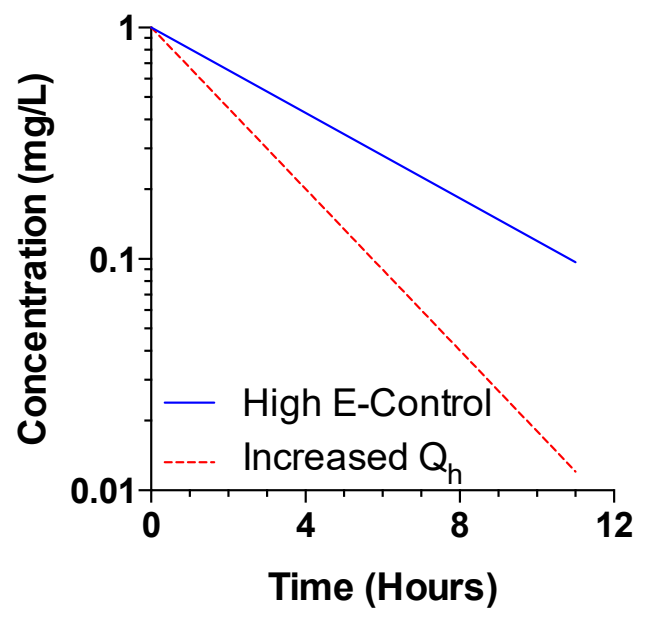

(B)

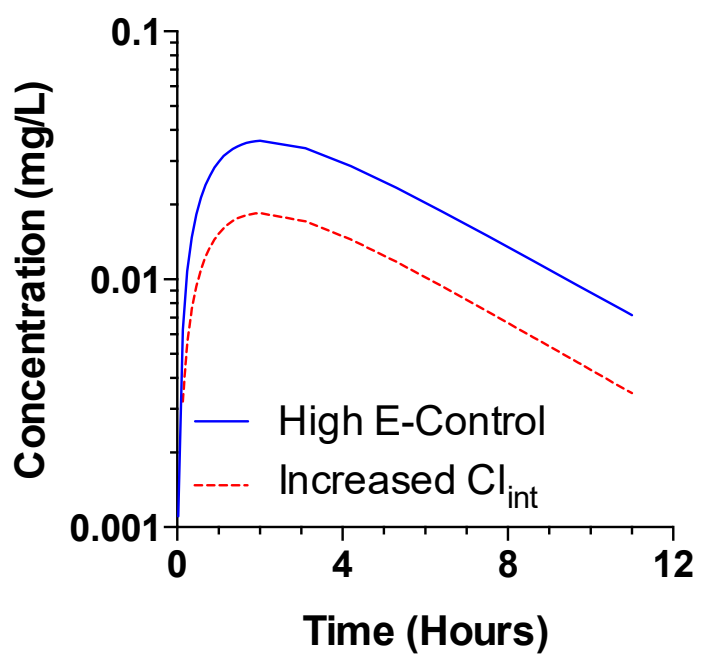

(D)

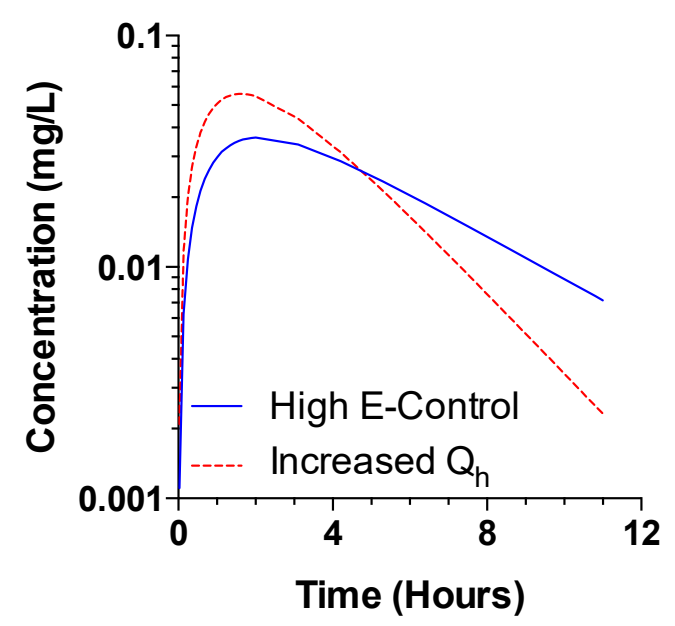

Figure 4. Effects of a two-fold increase in $C l_{i n t}\left(f_{u b}\right.$ or $\left.C l^{\prime}{ }_{i n t}\right)$ (top panels) or $Q_{h}$ (bottom panels) on the blood concentrationtime courses of a hypothetical high $E_{h}$ drug after intravenous (left) or oral (right) administration of a 400-mg dose of the drug. The kinetic parameters are listed in Table 2.

An example of the effects of changes in the blood flow on the pharmacokinetics in the literature is related to the interaction of lidocaine, a high $E_{h}$ drug, with metoprolol and propranolol, which reduce the cardiac output and hepatic blood flow (22). Lidocaine was administered by a short intravenous infusion alone or 1 day after pretreatment with metoprolol (50 mg orally every $6 \mathrm{~h}$ ) or propranolol ( $40 \mathrm{mg}$ orally every $6 \mathrm{~h}$ ). Metoprolol and propranolol reduced the clearance of intravenous lidocaine by $30 \%$ and $50 \%$ respectively, presumably due to their effects on the hepatic blood flow.
It should be noted that the changes in the clearance, in addition to affecting the half-life of the drug, also affects the maximum plasma concentration $\left(C_{\max }\right)$ and the time to reach $C_{\max }$ for orally-administered drug.

\section{Oral Clearance and Intrinsic Clearance}

The well-stirred model predicts that the oral clearance $\left(\mathrm{Cl}_{0}\right.$ or $\left.\mathrm{Cl} / \mathrm{F}\right)$ of a drug with hepatic elimination alone and no loss in the gastrointestinal $\operatorname{tract}\left(F=F_{h}\right)$ is in fact equal to its $C l_{\text {int }}\left(f_{u b} \cdot C l_{\text {int }}^{\prime}\right)$, therefore, being independent of $Q_{h}$ regardless of drug's $E_{h}$ : 


$$
\begin{aligned}
C l_{O}=\frac{\text { Dose }_{p o}}{A U C_{p o}} & =\frac{C l}{F}=\frac{Q_{h} \times E_{h}}{F_{h}}=\frac{Q_{h} \times \frac{C l_{i n t}}{Q_{h}+C l_{i n t}}}{\frac{Q_{h}}{Q_{h}+C l_{\text {int }}}}= \\
C l_{\text {int }} & =f_{u b} \cdot C l_{\text {int }}^{\prime}
\end{aligned}
$$

Consequently, it is not surprising that the oral AUC is not affected by $Q_{h}$, as shown in Figs. 3D and 4D for the low and high $E_{h}$ drugs, respectively. Even if the $F_{a b}$ and/or $F_{g}$ values are significantly lower than $1, C l_{o}$ is still a function of $C l_{\text {int }}$ (and not $Q_{h}$ ) as shown below:

$$
\begin{gathered}
C l_{O}=\frac{\text { Dose }_{p o}}{A U C_{p o}}=\frac{C l}{F}=\frac{Q_{h} \times E_{h}}{F_{a b} \times F_{g} \times F_{h}}=\frac{Q_{h} \times \frac{C l_{i n t}}{Q_{h}+C l_{\text {int }}}}{F_{a b} \times F_{g} \times \frac{Q_{h}}{Q_{h}+C l_{i n t}}}= \\
\frac{C l_{\text {int }}}{F_{a b} \times F_{g}}
\end{gathered}
$$

\section{EXAMPLES OF APPLICATION OF CLEARNCE CONCEPTS IN INTERPRETATION OF PHARMACOKINETIC BEHAVIOR OF DRUGS}

\section{Estimation of Hepatic Extraction Ratio of Drugs from In Vivo Pharmacokinetic Data}

Equation 3 may be rearranged to estimate $E_{h}$ of a drug after its intravenous administration:

$$
E_{h}=\frac{C l_{h}}{Q_{h}}
$$

After intravenous administration of the drug, the systemic $\mathrm{Cl}$ is estimated from the blood concentration-time data, and an estimate of $\mathrm{Cl}_{h}$ is obtained by either assuming $C l_{h}$ is equal to systemic clearance (i.e., for drugs with hepatic elimination only) or by subtracting renal $\mathrm{Cl}$ from the systemic clearance (i.e., for drugs with both renal and hepatic elimination), as discussed in detail recently (23). An estimate of $E_{h}$ is then obtained using the calculated $C l_{h}$ and an average $Q_{h}$. The estimates of $E_{h}$ value can then be used to predict the pharmacokinetics and blood concentration-time profiles of the drug in the presence of changes in the physiologic determinants of clearance such as organ blood flow, intrinsic clearance, or $f_{u b}$. The assumptions inherent to this type of calculation and the ramifications of using plasma or serum concentrations, instead of blood concentrations, and/or liver plasma flow, instead of liver blood flow, were recently discussed in detail (23). Briefly, it was shown that if plasma clearance and hepatic blood flow are used, the estimated $E_{h}$ would be accurate only if the blood: plasma concentration (B:P) ratio of the drug is equal to 1 . However, the use of the plasma concentrations and plasma liver flow will always result in an overestimation of the true $E_{h}$ unless the drug does not penetrate the red blood cells (23).

\section{Prediction of Oral Bioavailability from Oral Data Only}

In some cases, administration of a drug by intravenous method is not feasible or an injectable dosage form is not available. Using the clearance concepts, and some assumptions, it is possible to obtain a rough estimate of the extent of oral bioavailability of drugs using oral data only. We reported (24) the oral clearance $\left(\mathrm{Cl}_{o}\right)$ of tetrabenazine, a drug used for the control of movement disorders, in four patients with tardive dyskinesia. The $\mathrm{Cl}_{o}$ values were estimated by dividing the oral dose by the AUC. We then used the following equation, which is based on Equations 10 and 26 to estimate $F_{h}$ in these patients, assuming a hepatic blood flow of $1.28(\mathrm{~L} / \mathrm{h}) / \mathrm{kg}$ and complete oral absorption and intestinal availability (i.e., $F=$ $\left.F_{h}\right)$ :

$$
F=F_{h}=\frac{Q_{h}}{Q_{h}+C l_{\text {int }}}=\frac{Q_{h}}{Q_{h}+C l_{o}}
$$

The estimated $F$ values were $0.05,0.08,0.07$, and 0.02 for patients with $C l_{o}$ values of 431, 252, 299, and $1090(\mathrm{ml} / \mathrm{min}) / \mathrm{kg}$, respectively (24). Based on these values, we suggested that the oral bioavailability $(F)$ of tetrabenazine in humans is very low. Indeed, the $F$ value obtained in another study (25) after the intravenous and oral administration of the drug to humans $(0.05)$ was very close to the values obtained from the oral data alone.

It should be noted that these calculations assume elimination by the liver alone (i.e., no renal or gastrointestinal clearance). However, a modified version of Equation 29 may be provided for drugs with both renal and hepatic clearance pathways, as shown below:

$$
\begin{gathered}
C l_{O}=\frac{C l}{F}=\frac{C l_{r}+C l_{h}}{F_{h}}=\frac{C l_{r}+Q_{h} \times E_{h}}{F_{h}}= \\
\frac{C l_{r}+Q_{h} \times\left(1-F_{h}\right)}{F_{h}}=\frac{C l_{r}+Q_{h}-Q_{h} F_{h}}{F_{h}} \\
C l_{O} \times F_{h}=C l_{r}+Q_{h}-Q_{h} F_{h} \\
F_{h}\left(C l_{O}+Q_{h}\right)=C l_{r}+Q_{h}
\end{gathered}
$$




$$
F_{h}=\frac{C l_{r}+Q_{h}}{Q_{h}+C l_{o}}
$$

After determination of $C l_{r}$ and $C l_{o}$, Equation 33 may be used to estimate $F_{h}$ (or $F$ ) for drugs with both hepatic and renal elimination.

\section{Differentiation of Gut and Hepatic First-Pass Metabolism of Drugs}

An application of clearance concept in the literature is for differentiation of first-pass metabolism in the gut from that in the liver. After oral administration, the oral bioavailability of a drug $(F)$ is a function of the fraction of the drug absorbed $\left(F_{a b}\right)$, fraction of the drug escaping metabolism in the gastrointestinal tract $\left(F_{g}\right)$, and fraction of the drug escaping the hepatic metabolism or hepatic availability $\left(F_{h}\right)$, as demonstrated in Fig. 2 and Equation 24. Once a drug is administered by both oral and intravenous routes, $F$ may be estimated from the oral and intravenous AUC values (correcting for the dose). Additionally, the hepatic $E_{h}$ and $F_{h}$ may be estimated from the intravenous data using Equations 28 and 4, respectively, assuming an average $Q_{h}$ of 1500 $\mathrm{mL} / \mathrm{min}$ in a $70-\mathrm{kg}$ subject. Consequently, the value of $F_{a b} \times F_{g}$ may be estimated from the $F$ and $F_{h}$ values by rearranging Equation 24:

$$
F_{a b} \times F_{g}=\frac{F}{F_{h}}
$$

This method allows one to separate the contribution of the liver $\left(F_{h}\right)$ from that of the gut $\left(F_{a b} \times F_{g}\right)$ to the overall loss of the drug after the oral administration. However, in the absence of any additional information about the extent of absorption of the drug, $F_{a b}$ and $F_{g}$ cannot be separated from each other.

An application of this method was demonstrated by Hebert et al. (26), who administered a single oral (10 $\mathrm{mg} / \mathrm{kg}$ ) or intravenous $(3 \mathrm{mg} / \mathrm{kg}$ ) dose of cyclosporine to six healthy subjects and found average blood AUC values of 8986 and 10092 ng.h $/ \mathrm{mL}$, respectively. Based on the oral and intravenous data, the oral bioavailability $(F)$ of cyclosporine in these patients was 0.27 :

$$
\begin{gathered}
F=\frac{A U C_{P O}}{A U C_{i v}} \times \frac{\text { Dose }_{i v}}{\text { Dose }_{p o}} \\
F=\frac{8986}{10092} \times \frac{3}{10}=0.27
\end{gathered}
$$

The systemic clearance of cyclosporine based on the intravenous dose of $3 \mathrm{mg} / \mathrm{kg}$ and $\mathrm{AUC}_{\mathrm{iv}}$ of 10092 was $0.3 \mathrm{~L} / \mathrm{kg}$ :

$$
C l=\frac{\text { Dose }_{i v}}{A U C_{i v}}=\frac{3000}{10092}=0.3 \mathrm{~L} / \mathrm{kg}
$$

Assuming the systemic clearance of cyclosporine is equal to its hepatic clearance (i.e., gut clearance doses not significantly contribute to the systemic clearance) and a $Q_{h}$ of $1.286 \mathrm{~L} / \mathrm{h} / \mathrm{kg}(90 \mathrm{~L} / \mathrm{h}$ in a $70-$ $\mathrm{kg}$ subject), the $E_{h}$ and $F_{h}$ values were then estimated:

$$
\begin{gathered}
E_{h}=\frac{0.3}{1.286}=0.23 \\
F_{h}=1-0.23=0.77
\end{gathered}
$$

Using the predicted $F_{h}$ and measured $F$, the $F_{a b} \times F_{g}$ value of cyclosporine was then determined:

$$
F_{a b} \times F_{g}=\frac{F}{F_{h}}=\frac{0.27}{0.77}=0.35
$$

A comparison of $F(0.27), F_{h}(0.77)$, and $F_{a b} \times F_{g}$ (0.35) values of cyclosporine in these patients, clearly suggests that the low oral bioavailability of the drug is mostly due to the loss of drug in the gastrointestinal tract than its loss during the first-pass metabolism in the liver. Later, Galetin et al. (27) used this method to estimate the $F_{g}$ of a number of drugs, with an assumption that the absorption of the drugs, if experimentally unknown, was complete $\left(F_{a b}=1\right)$.

\section{Differentiation of Incomplete Absorption from the First-Pass Metabolism in the Gut and Liver}

Wu et al. (19) proposed a method to determine $F_{a b}$, $F_{g}$, and $F_{h}$ separately, based on the intravenous and oral administration of a drug in the absence and presence of interacting drugs known to inhibit its metabolism. If the interacting and victim drugs are administered several hours apart from each other, one may assume that the interacting drug would only affect the metabolism and not the extent of absorption of the victim drug. In that case, the following equations may be defined:

$$
F=F_{a b} \times F_{g} \times F_{h}
$$

In the Absence of Interaction

$$
F_{i t x}=F_{a b} \times F_{g, i t x} \times F_{h, i t x}
$$

In the Presence of Interaction 
where the subscript itx indicates the parameter in the presence of the interacting drug. The values of $F_{a b} \times F_{g}$ or $F_{a b} \times F_{g, i t x}$ may be easily estimated from $F$ and $F_{h}$ or $F_{i t x}$ and $F_{h, i t x}$, respectively, using Equation 34.

In the case of an enzyme inhibitor interaction, the value of $F_{a b}$ may be estimated between its two minimum and maximum boundaries. The maximum boundary of $F_{a b}$ is 1 . The minimum boundary of $F_{a b}$ is assuming $F_{g, i t x}$ is equal to 1 (i.e., complete inhibition of gut metabolism). Setting $F_{a b}$ to its maximum (i.e., 1) or minimum (calculated from the enzyme inhibition data) boundaries, the values of $F_{g}$ in control subjects (i.e., the absence of drug interaction) may be estimated between a minimum and a maximum.

The method was used to determine the contribution of hepatic and gastrointestinal first-pass metabolism to the overall $F$ of cyclosporine in the presence and absence of two enzyme inhibitors (erythromycin and ketoconazole) and an enzyme inducer (rifampin) (19). Here, we present the data (28) and calculations (19) for the ketoconazole inhibition study, which was conducted in five healthy subjects. The doses and average blood AUC values after the oral and intravenous administration of cyclosporine in the absence (Control) and presence of ketoconazole are listed in Table 3 (28).

Using the average values presented in Table 3 , The $F, F_{h}$, and $F_{a b} \times F_{g}$ values in Control subjects may be estimated as shown below:

$$
\begin{gathered}
F=\frac{A U C_{P O}}{A U C_{i v}} \times \frac{\text { Dose }_{i v}}{\text { Dose }_{p o}}=\frac{5938}{6683} \times \frac{0.5}{2}=0.222 \\
C l=\frac{\text { Dose }_{i v}}{A U C_{i v}}=\frac{2000}{6683}=0.299 \mathrm{~L} / \mathrm{h} / \mathrm{kg} \\
F_{h}=1-E_{h}=1-\frac{C l_{h}}{Q_{h}}=1-\frac{0.299}{1.28}=1-0.234 \\
=0.766 \\
F_{a b} \times F_{g}=\frac{F}{F_{h}}=\frac{0.222}{0.766}=0.290
\end{gathered}
$$

Similarly, $F_{i t x}, F_{h, i t x}$, and $F_{a b} \times F_{g, i t x}$ in the presence of ketoconazole may be calculated using the respective data in Table 3 . The summary of the above calculations, based on the average AUC value reported before (28), are presented in Table 4.
The data in the presence of ketoconazole, presented in Table 4, suggest that two boundaries of $F_{a b}$ are 1 and 0.722 . Consequently, $F_{g}$ may be estimated at these two boundaries:

$$
\begin{gathered}
F_{a b} \times F_{g}=0.290 \\
F_{g}=\frac{0.290}{F_{a b}} \\
F_{g-\text { low }}=\frac{0.290}{1}=0.290 \\
F_{\text {g-upp }}=\frac{0.290}{0.722}=0.402
\end{gathered}
$$

Therefore, the lower $\left(F_{g \text {-low }}\right)$ and upper $\left(F_{g-u p p}\right)$ limits of $F_{g}$ for cyclosporine in healthy volunteers are 0.290 and 0.402 , whereas the lower and upper limits of $F_{a b}$ are 0.722 and 1, respectively. Considering an $F_{h}$ of 0.766 (Table 4), the data suggest that the low oral bioavailability of cyclosporine in Control subjects (0.222) is indeed mostly due to its first-pass metabolism in the gut $\left(F_{g}\right.$ of 0.290-0.402), whereas the oral absorption of the formulation is relatively high ( $F_{a b}$ of $0.722-1.0$ ). Again, these calculations assume that gut clearance, although reducing the presystemic availability of the drug, does not significantly contribute to the systemic clearance of the drug, meaning that $\mathrm{Cl}$ is equal to $\mathrm{Cl}_{h}$.

More recently, Hisaka et al. (29) developed a new method based on the concept of drug-drug interactions to estimate $F_{g}$ values from the changes in the AUC and half-life values of the victim drug in the presence of the perpetrating drug. The method is based on the idea that the inhibition of intestinal metabolism causes a change in the AUC without any changes in the plasma half-life. However, inhibition of hepatic metabolism results in changes in both halflife and AUC, unless the clearance of the drug is flow-limited (i.e., drugs with high extraction ratio). Nevertheless, the method still requires an approximation of $F_{a b}$ value. Using this method, the authors estimated $F_{g}$ values of $0.56 \pm 0.29$ (mean \pm SD) for 20 drugs that were CYP3A substrates and $0.86 \pm 0.11$ for 8 drugs that were not CYP3A substrates. The interested readers are referred to the original article (29) for details about the method and derivation of equations. 
Table 3. The dose and average AUC values of cyclosporine after intravenous and oral administration of the drug to healthy subjects in the absence (Control) and presence of ketoconazole ${ }^{\mathrm{a}}$

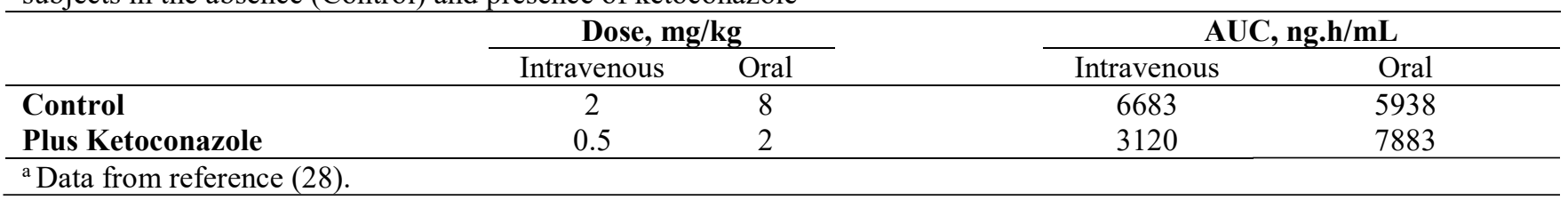

Table 4. The calculated oral bioavailability $(F)$ and estimated hepatic availability $\left(F_{h}\right)$ and products of fraction absorbed $\left(F_{a b}\right)$ and gut availability $\left(F_{g}\right)$ of cyclosporine in the absence (Control) and presence of ketoconazole ${ }^{\mathrm{a}}$

\begin{tabular}{lccc}
\hline & $\boldsymbol{F}$ & $\boldsymbol{F}_{\boldsymbol{h}}$ & $\boldsymbol{F}_{\boldsymbol{a b}} \boldsymbol{.} \boldsymbol{F}_{\boldsymbol{g}}$ \\
\hline Control & 0.222 & 0.766 & 0.290 \\
Plus Ketoconazole & 0.632 & 0.875 & 0.722 \\
\hline${ }^{\mathrm{a}}$ Calculations are based on the average AUC values and doses presented in Table 3. & & & \\
\hline
\end{tabular}

\section{Estimation of Gastrointestinal Availability from Grapefruit Juice-Drug Interaction Studies}

Grapefruit juice (GFJ) components are believed to irreversibly inhibit intestinal CYP3A4, without any major effect on the liver CYP3A4 (30). Therefore, co-administration of grapefruit juice with an orallyadministered drug that is subject to intestinal CYP3A4 metabolism inhibits the drug's CYP3A4mediated, presystemic intestinal metabolism. Gertz et al. (31) used available data in the literature related to the interaction of drugs with grapefruit juice to estimate their $F_{g}$, using the following equation:

$$
F_{g}=\frac{A U C_{\text {Control }}}{A U C_{G F J}}
$$

where $A U C_{\text {Control }}$ and $A U C_{G F J}$ are the AUC of the drug in the absence and presence of GFJ administration, respectively. This method assumes complete intestinal metabolism of the drug through CYP3A4, complete inhibition of intestinal metabolism with GFJ, and no effect of GFJ on the systemic (liver) metabolism or clearance or the extent of absorption $\left(F_{a b}\right)$ of the drug. Additionally, the authors (31) suggested that the method's applicability to drugs that are subject to transport or efflux in the gastrointestinal tract is limited.

\section{LIMITATIONS}

It should be noted that while clearance concepts are very useful in predicting the pharmacokinetic behavior of drugs, their applications to estimation of $F$ and its components $\left(F_{a b}, F_{g}\right.$, and $\left.F_{h}\right)$ in different situations presented here are associated with a number of assumptions, hence subject to some degree of error. First, the AUC and clearance terms in the above equations are based on the blood concentrations of the drug. Therefore, the use of plasma values, which are commonly measured, may introduce substantial errors in the estimation of the kinetic parameters (23). Second, a number of calculations listed above use an average liver blood flow (e.g., $1500 \mathrm{~mL} / \mathrm{min}$ ), which might be different than the actual $Q_{h}$ in the studied subjects. This is because although intra- and inter-individual variabilities in the hepatic blood flow in healthy subjects under controlled conditions appears to be relatively low (32-34), physiological factors (e.g., posture and exercise), pharmacological interventions (e.g., beta-blockers), disease states (e.g., heart failure), or food ingestion may significantly affect hepatic blood flow $(35,36)$. Other assumptions include linear pharmacokinetics and a constant systemic clearance after different routes of administration. With specific reference to methods estimating $F_{g}$, additional assumptions are that the intestinal clearance, although reducing the presystemic availability of the drug, does not significantly contribute to the systemic clearance of the drug. Lastly, estimation of $F_{g}$ is also based on the assumption that the drug is not subject to influx or efflux transporters. Nevertheless, despite these assumptions and/or limitations, application of clearance concepts has resulted in substantial improvement in our understanding of the pharmacokinetics of many drugs. 


\section{CONCLUSIONS}

In conclusion, development of clearance concepts has significantly moved the discipline of pharmacokinetics forward. Although clearance concepts are more discussed in terms of hepatic clearance, they are equally applicable to clearance by other eliminating organs, such as the kidneys. The concepts allow definition of organ and total clearance values in terms of physiologic parameters such as organ blood flow and intrinsic capability of the eliminating organ to remove the drug from the body. Furthermore, the clearance concepts allow prediction of the pharmacokinetics of drugs after intra- and extravascular routes in the presence of disease states, drug interactions, or as a result of patients' age. Researchers have applied these concepts to predict the oral bioavailability of drugs in the absence of otherwise needed intravenous data, to distinguish between the role of liver and gut in the first pass metabolism of drugs, and to differentiate between the incomplete oral absorption and metabolism in the gut after oral administration of drugs. More than 45 years after their introduction, clearance concepts remain a powerful tool in explaining the pharmacokinetics of drugs and predicting the changes in the blood concentrationtime course of drugs when the underlying physiologic parameters are altered.

\section{REFERENCES}

1. Gillette JR. Factors affecting drug metabolism. Ann N Y Acad Sci. 1971;179:43-66.

2. Rowland M, Benet, L.Z., Grahm, G.G. Clearance concepts in pharmacokinetics. J Pharmacokinet Biopharm. 1973;1:123-36.

3. Wilkinson G, Shand D. A physiological approach to hepatic drug clearance. Clin Pharmacol Ther. 1975;18:377-89.

4. Benet LZ. Clearance (nee Rowland) concepts: a downdate and an update. J Pharmacokinet Pharmacodyn. 2010;37:529-39.

5. Mehvar R. Interdependency of pharmacokinetic parameters: a chicken-and-egg problem? Not! J Pharm Pharm Sci. 2006;9:113-8.

6. Sirianni GL, Pang KS. Organ clearance concepts: New perspectives on old principles. J Pharmacokinet Biopharm. 1997;25:449-70.

7. Pang KS, Weiss M, Macheras P. Advanced pharmacokinetic models based on organ clearance, circulatory, and fractal concepts. AAPS J. 2007;9:E268-83.
8. Pang K, Rowland M. Hepatic clearance of drugs. I. Theoretical considerations of a "well-stirred" model and a "parallel tube" model. Influence of hepatic blood flow, plasma and blood cell binding, and the hepatocellular enzymatic activity on hepatic drug clearance. J Pharmacokinet Biopharm. 1977;5:62553.

9. Pang K, Rowland M. Hepatic clearance of drugs. II. Experimental evidence for acceptance of the "wellstirred" model over the "parallel tube" model using lidocaine in the perfused rat liver in situ preparation. J Pharmacokinet Biopharm. 1977;5:655-79.

10. Pang K, Rowland M. Hepatic clearance of drugs. III. Additional experimental evidence supporting the "well-stirred" model, using metabolite (MEGX) generated from lidocaine under varying hepatic blood flow rates and linear conditions in the perfused rat liver in situ preparation. J Pharmacokinet Biopharm. 1977;5:681-99.

11. Roberts M, Rowland M. Hepatic elimination-dispersion model. J Pharm Sci. 1985;74:585-7.

12. Gray M, Tam Y. The series-compartment model for hepatic elimination. Drug Metab Disposit. 1986;15:27-31.

13. Pang KS, Terrell JA, Nelson SD, Feuer KF, Clements MJ, Endrenyi L. An enzyme-distributed system for lidocaine metabolism in the perfused rat liver preparation. J Pharmacokinet Biopharm. 1986;14:107-30.

14. Bass L, Robinson P, Bracken AJ. Hepatic elimination of flowing substrates: the distributed model. J Theor Biol. 1978;72:161-84.

15. Goresky CA, Bach GG, Nadeau BE. On the uptake of materials by the intact liver. The transport and net removal of galactose. J Clin Invest. 1973;52:9911009.

16. Yamazaki M, Akiyama S, Nishigaki R, Sugiyama Y. Uptake is the rate-limiting step in the overall hepatic elimination of pravastatin at steady state in rats. Pharm Res. 1996;13:1559-64.

17. Benet LZ, Liu S, Wolfe AR. The universally unrecognized assumption in predicting drug clearance and organ extraction ratio. Clin Pharmacol Ther. 2018;103:521-5.

18. Rowland M, Pang KS. Commentary on "The universally unrecognized assumption in predicting drug clearance and organ extraction ratio". Clin Pharmacol Ther. 2018;103:386-8.

19. Wu CY, Benet LZ, Hebert MF, Gupta SK, Rowland M, Gomez DY, et al. Differentiation of absorption and first-pass gut and hepatic metabolism in humans: Studies with cyclosporine. Clin Pharmacol Ther. 1995;58:492-7.

20. O'Reilly RA. Interaction of sodium warfarin and rifampin. Studies in man. Ann Intern Med. 1974;81:337-40. 
21. Alvan G, Piafsky K, Lind M, von Bahr C. Effect of pentobarbital on the disposition of alprenolol. Clin Pharmacol Ther. 1977;22:316-21.

22. Conrad KA, Byers JM, 3rd, Finley PR, Burnham L. Lidocaine elimination: effects of metoprolol and of propranolol. Clin Pharmacol Ther. 1983;33:133-8.

23. Mehvar R. Application of organ clearance to estimation of the in vivo hepatic extraction ratio. Curr Clin Pharmacol. 2016;11:47-52.

24. Mehvar R, Jamali F, Watson M, Skelton D. Pharmacokinetics of tetrabenazine and its major metabolite in man and rat: bioavailability and dose dependency studies. Drug Metab Disposit. 1987;15:250-5.

25. Roberts MS, McLean S, Millingen KS, Galloway HM. The pharmacokinetics of tetrabenazine and its hydroxy metabolite in patients treated for involuntary movement disorders. Eur J Clin Pharmacol. 1986;29:703-8.

26. Hebert MF, Roberts JP, Prueksaritanont T, Benet LZ. Bioavailability of cyclosporine with concomitant rifampin administration is markedly less than predicted by hepatic enzyme induction. Clin Pharmacol Ther. 1992;52:453-7.

27. Galetin A, Gertz M, Houston JB. Potential role of intestinal first-pass metabolism in the prediction of drug-drug interactions. Expert Opin Drug Metab Toxicol. 2008;4:909-22.

28. Gomez DY, Wacher VJ, Tomlanovich SJ, Hebert MF, Benet LZ. The effects of ketoconazole on the intestinal metabolism and bioavailability of cyclosporine. Clin Pharmacol Ther. 1995;58:15-9.
29. Hisaka A, Nakamura M, Tsukihashi A, Koh S, Suzuki H. Assessment of intestinal availability (FG) of substrate drugs of cytochrome p450s by analyzing changes in pharmacokinetic properties caused by drug-drug interactions. Drug Metab Dispos. 2014;42:1640-5.

30. Bailey DG, Malcolm J, Arnold O, Spence JD. Grapefruit juice-drug interactions. $\mathrm{Br} \quad \mathrm{J} \quad \mathrm{Clin}$ Pharmacol. 1998;46:101-10.

31. Gertz M, Davis JD, Harrison A, Houston JB, Galetin A. Grapefruit juice-drug interaction studies as a method to assess the extent of intestinal availability: utility and limitations. Curr Drug Metab. 2008;9:78595.

32. Soons PA, De Boer A, Cohen AF, Breimer DD. Assessment of hepatic blood flow in healthy subjects by continuous infusion of indocyanine green. $\mathrm{Br} \mathrm{J}$ Clin Pharmacol. 1991;32:697-704.

33. Richardson PD, Withrington PG. Liver blood flow. I. Intrinsic and nervous control of liver blood flow. Gastroenterology. 1981;81:159-73.

34. Carlisle KM, Halliwell M, Read AE, Wells PN. Estimation of total hepatic blood flow by duplex ultrasound. Gut. 1992;33:92-7.

35. Daneshmend TK, Jackson L, Roberts CJ. Physiological and pharmacological variability in estimated hepatic blood flow in man. Br J Clin Pharmacol. 1981;11:491-6.

36. McLean AJ, McNamara PJ, duSouich P, Gibaldi M, Lalka D. Food, splanchnic blood flow, and bioavailability of drugs subject to first-pass metabolism. Clin Pharmacol Ther. 1978;July:5-10. 